Review Article

\title{
The Triggering Receptor Expressed on Myeloid Cells 2: "TREM-ming" the Inflammatory Component Associated with Alzheimer's Disease
}

\author{
Troy T. Rohn \\ Department of Biological Sciences, Boise State University, Science Building, Room 228, Boise, ID 83725, USA \\ Correspondence should be addressed to Troy T. Rohn; trohn@boisestate.edu
}

Received 6 December 2012; Accepted 7 February 2013

Academic Editor: Emilio Luiz Streck

Copyright (C) 2013 Troy T. Rohn. This is an open access article distributed under the Creative Commons Attribution License, which permits unrestricted use, distribution, and reproduction in any medium, provided the original work is properly cited.

\begin{abstract}
Alzheimer's disease (AD) is an age-related neurodegenerative disorder characterized by a progressive loss of memory and cognitive skills. Although much attention has been devoted concerning the contribution of the microscopic lesions, senile plaques, and neurofibrillary tangles to the disease process, inflammation has long been suspected to play a major role in the etiology of AD. Recently, a novel variant in the gene encoding the triggering receptor expressed on myeloid cells 2 (TREM2) has been identified that has refocused the spotlight back onto inflammation as a major contributing factor in AD. Variants in TREM2 triple one's risk of developing late-onset AD. TREM2 is expressed on microglial cells, the resident macrophages in the CNS, and functions to stimulate phagocytosis on one hand and to suppress cytokine production and inflammation on the other hand. The purpose of this paper is to discuss these recent developments including the potential role that TREM2 normally plays and how loss of function may contribute to $\mathrm{AD}$ pathogenesis by enhancing oxidative stress and inflammation within the CNS. In this context, an overview of the pathways linking beta-amyloid, neurofibrillary tangles (NFTs), oxidative stress, and inflammation will be discussed.
\end{abstract}

\section{Defining Alzheimer's Disease}

Alzheimer's disease $(\mathrm{AD})$ is classified as a neurodegenerative disorder affecting neurons of the brain that are responsible for memory and higher cognitive functions. The brain consists of over a 100-billion neurons that specialize in the ability to transmit information to other cells, and thus constitute the basic working unit of the brain. Because cortical neurons, in general, do not have the capacity to regenerate, once neurons are lost and symptoms manifest, the process is essentially irreversible. In this manner, Alzheimer's is classified as a progressive neurodegenerative disease that can take anywhere from 5-20 years to run its course. The loss of these neurons is significant with affected individuals losing up to $50 \%$ mass of the brain over the course of the disease. The loss of these neurons leads to the symptoms of the disease including memory impairments, difficulties with language, inability to execute motor activities, and the overall decline in cognitive skills [1]. Dementia is the umbrella term describing the symptoms of $\mathrm{AD}$, and $\mathrm{AD}$ is by far the leading cause of dementia in the United States, being responsible for over 70\% of all known cases of dementia [2]. AD is a multifactorial disorder, whose causes remain largely unknown. Despite extensive research on genetic factors, the vast majority of Alzheimer's cases $(>90 \%)$ are not directly linked to them [3]. Aging is the most well-established risk factor for the development of sporadic $\mathrm{AD}$ with incidence rates showing an exponential growth between the ages of 65 and 85 years, doubling every 5 years [3].

The national numbers on $\mathrm{AD}$ are alarming: currently one in eight older Americans has AD making it the sixth leading cause of death in the United States. An estimated 5.4 million Americans have $\mathrm{AD}$, a figure that includes 5.2 million people age 65 and older [1]. Of those with $\mathrm{AD}$, an estimated 4 percent are under the age 65,6 percent are 64 to 74,44 percent are 75 to 84 , and 46 percent are 85 or older [1]. Of all of the major causes of death in the United States, including stroke, cancer, and heart disease, only Alzheimer's disease has shown a significant increase in mortality during the same time frame (2000-2008). 


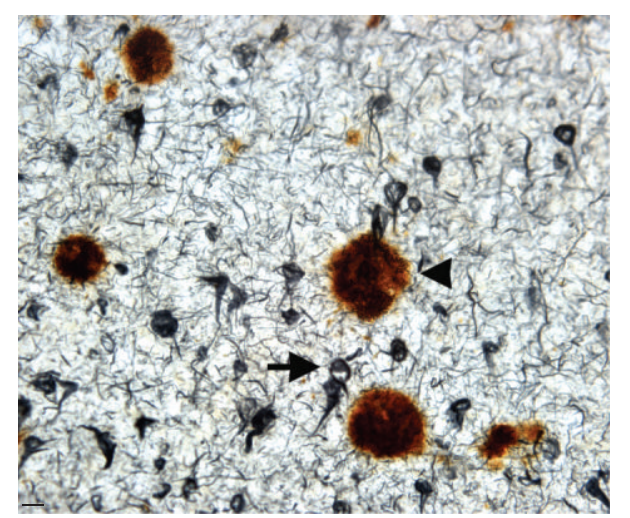

(a)

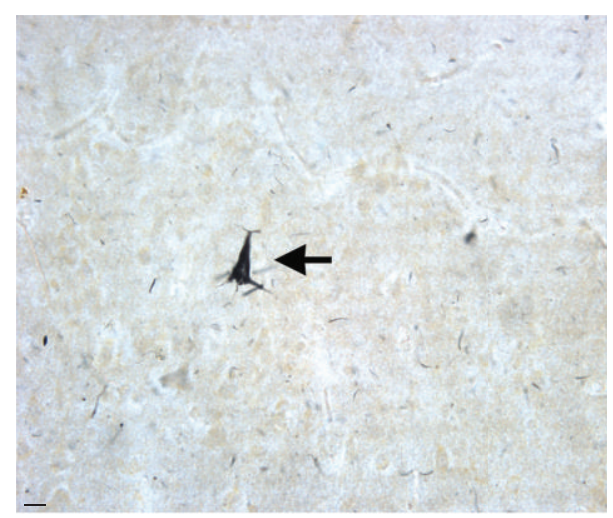

(b)

FIGURE 1: The microscopic trouble makers in Alzheimer's disease: senile plaques and neurofibrillary tangles. (a) In AD, widespread accumulation of extracellular beta-amyloid plaques is evident (arrowhead), together with the presence of an abundance of NFTs along with neuropil threads, which are composed principally of modified and aggregated tau (arrow). (b) For comparison purposes, an age-matched control brain is depicted indicating a complete absence of plaques. However, it is not uncommon to find an occasional tangle (arrow) in the normally aged brain although the numbers of tangles is minimal by comparison. Brain sections are representative staining of the hippocampus using an anti-beta-amyloid antibody to label plaques (brown) and anti-PHF-1 (black) to label NFTs. Scale bars are $10 \mu \mathrm{m}$.

\section{Pathology Associated with AD}

$\mathrm{AD}$ is diagnosed based upon the extent of senile plaques composed of beta-amyloid and neurofibrillary tangles (NFTs) containing abnormally phosphorylated and truncated tau [4]. The preponderance of research to date suggest a pivotal role for beta-amyloid in the progression of $\mathrm{AD}$, and collectively this concept has coined the beta-amyloid hypothesis [5]. In essence, this hypothesis stipulates that much of the pathology associated with $\mathrm{AD}$ is driven by an increased load of betaamyloid in the brain of $\mathrm{AD}$ patients that can occur years before the first symptoms of the disease manifest.

Beta-amyloid is formed following sequential cleavage of the amyloid precursor protein (APP) by two proteases, $\beta$ secretase and $\gamma$-secretase. Once formed, beta-amyloid has the propensity to self-aggregate into $\beta$-sheet structures that deposit extracellularly forming senile plaques (Figure 1). More recently, the beta-amyloid hypothesis has been modified to the "toxic beta-amyloid oligomer" hypothesis to reconcile the apparent lack of correlation between beta-amyloid in plaques and cognitive impairment [6]. This reformulation of the amyloid cascade hypothesis focuses on oligomeric aggregates of beta-amyloid as the prime toxic species causing $\mathrm{AD}$ in part because this form of beta-amyloid strongly correlates with the severity of dementia $[7,8]$. In addition, this oligomeric form of beta-amyloid is highly toxic and is the trigger for the loss of synapses and neuronal damage $[9,10]$. Given the strong support for the amyloid cascade hypothesis, many of the current therapeutic strategies now in clinical trials involve some aspect of modifying beta-amyloid production or clearance [11]. However, despite the overwhelming evidence supporting a role for beta-amyloid in $\mathrm{AD}$, this hypothesis is currently under critical assessment due to the recent clinical trial failures based on the strategy of lowering the beta-amyloid levels in the AD brain [12]. For example, one strategy currently being investigated involves inhibiting gamma-secretase to limit the production of the betaamyloid peptide derived from APP. One such compound, semagacestat, showed promise in early clinical trials, but a recent phase III trial involving over 2,600 participants was discontinued after failure to demonstrate efficacy. Compared to placebo, patients receiving semagacestat actually did worse both in daily function and cognition and were at higher risk of developing skin cancer [13].

The other major pathological finding in $\mathrm{AD}$ is the presence of neurofibrillary tangles (NFTs) (Figure 1) [14]. NFTs are primarily composed of aggregated phosphorylated tau protein and are a clinical feature not just in $\mathrm{AD}$ but other diseases that are collectively referred to as "tauopathies" [15]. Tau normally functions to help maintain the stability of the cytoskeleton of neurons by binding to microtubules. However, upon hyperphosphorylation and posttranslational cleavage, tau loses its binding affinity for microtubules, leading to a destabilization of the cytoskeleton and self-assembly of tau into tangles of paired helical filaments (PHFs) [16]. Although not universally accepted, it has been proposed that NFTs may not be a central mediator of disease pathogenesis, but instead, NFTs may actually serve a protective rather than harmful function by providing a compensatory response mounted by neurons against oxidative stress [17].

\section{Mechanisms of Neurodegeneration in AD}

According to the beta-amyloid hypothesis, the accumulation and aggregation of beta-amyloid into toxic soluble oligomers is the first step leading to neuronal degeneration in $\mathrm{AD}$ [18]. Specifically, an important early molecular step is the lost of synapses, which correlates highly with the initial memory impairment observed in $\operatorname{AD}[19,20]$. Intensive research over the last two decades has examined potential pathways activated by beta-amyloid aggregates that lead to synaptic dysfunction, NFT formation, and eventual cell death. Figure 2 summarizes some of the major findings on beta-amyloid-induced toxicity that begin with either betaamyloid activation of apoptotic pathways or promotion of 


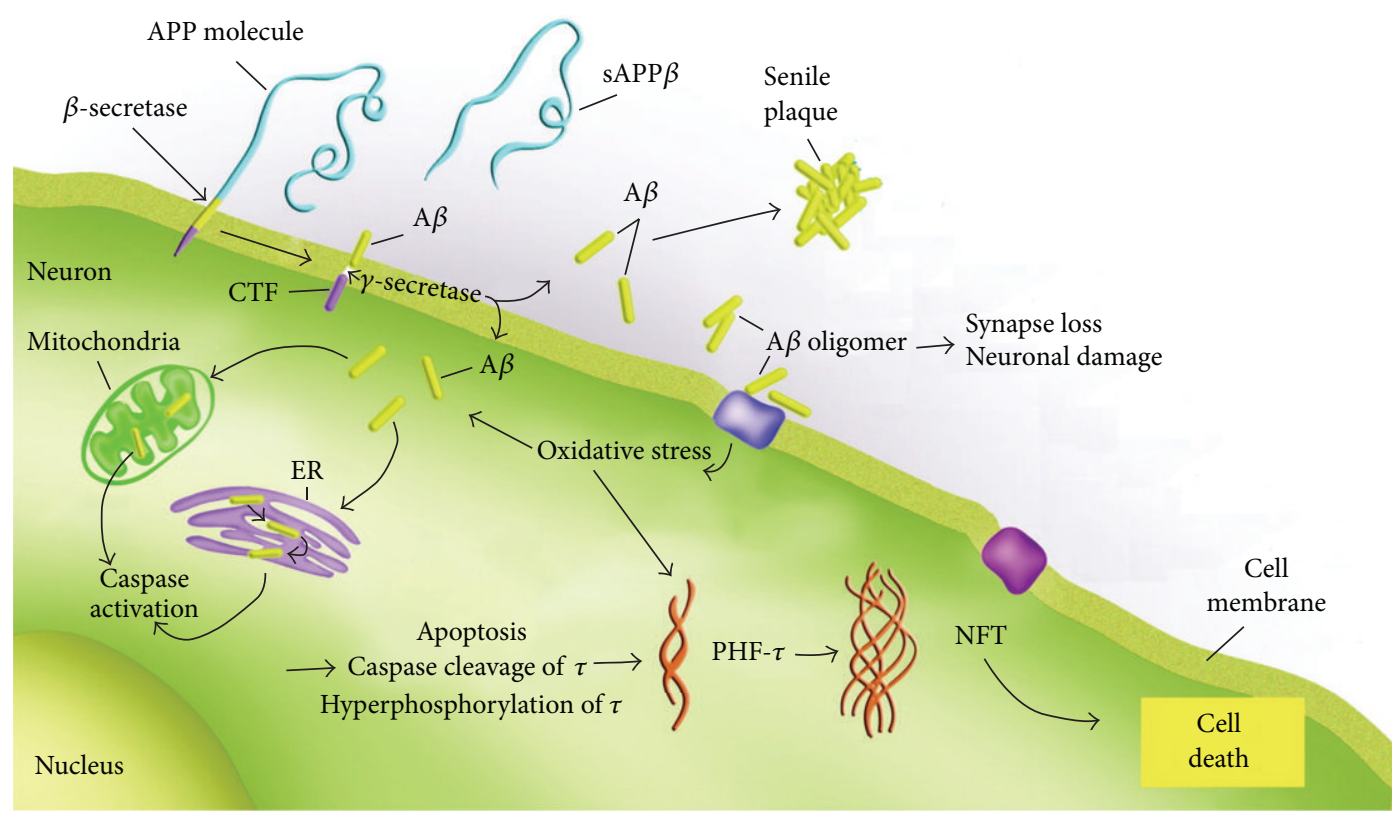

FIGURE 2: Putative pathway for neurodegeneration in Alzheimer's disease. According to the beta-amyloid hypothesis, the production of betaamyloid represents the first step in the entire process following the sequential cleavage of APP by $\beta$-secretase and $\gamma$-secretase. Beta-amyloid in turn may lead to NFT formation and eventual cell death by promoting oxidative stress and caspase activation through initiation of the mitochondrial-mediated pathway of apoptosis. The activation of caspases results in cleavage of critical cellular proteins including tau, leading to its modification and hyperphosphorylation, a key prerequisite for filament formation. See main text for details.

oxidative stress. With regards to apoptosis, since the early nineties, studies have supported a general role for apoptosis in $\mathrm{AD}$ [21-23]. In addition, the activation of caspases, including caspase $3,6,8$, and 9 , has been documented in the AD brain [24-30]. In turn, evidence suggests that once activated, caspases may cleave critical cellular proteins in $\mathrm{AD}$ including APP, actin, fodrin, glial acidic fibrillary protein, beclin-1, and tau [28, 31-35]. Importantly, several studies have suggested that caspase activation and cleavage of tau may precede and contribute to the formation of NFTs $[31,32,36]$.

An interlinking step between beta-amyloid and NFTs could be the promotion of oxidative stress. Oxidative stress either through lipid peroxidation or mitochondrial disruption is an early feature found in $\mathrm{AD}$ [37-39]. In addition, oxidative stress may contribute to the activation of apoptosis through both the extrinsic and intrinsic pathway [40, 41]. Finally, tau phosphorylation is upregulated by oxidative stress [42] and tau filaments are modified by products of oxidative stress [43-45]. Oxidative stress also activates several kinases that have been implicated in the hyperphosphorylation of tau including glycogen synthase kinase-3 (GSK3), Jun-N-terminal kinase (JNK), and mitogen-activated protein kinases (MAPKs) [46, 47].

\section{Inflammation in AD}

Brain inflammation is a pathological hallmark of $\mathrm{AD}[48,49]$. In this regard, numerous studies have supported a definitive role for inflammation in $\mathrm{AD}$, with a key feature being the presence of activated microglia [50-52] and reactive astrocytes found within senile plaques [53-55]. Epidemiological studies have also pointed to inflammation as central to $\mathrm{AD}$, indicating that the long-term use of anti-inflammatory drugs is linked with reduced risk of developing the disease [56]. Microglia are key players in mediating immune responses in the CNS functioning as the resident macrophages of the CNS and as such contribute to a healthy CNS by attacking and removing potential pathogens and cell debris and by secreting tissue rebuilding factors [57].

Interestingly, the link between the activation of microglia and inflammation may be beta-amyloid. Thus, beta-amyloid is a potent inducer of microglia activation $[52,58-60]$, and one important role of microglia is to clear beta-amyloid deposits out of the $\mathrm{AD}$ brain (for recent review, see [61]).

One potential caveat with inflammation is determining cause and effect. Does AD cause inflammation? does the dysregulation of immune system pathways trigger the disease process? Alternatively, although chronic inflammation may be a driving force in disease pathogenesis, it also may serve as a beneficial response at least early on during the course of AD. Finally, it is possible that inflammation could simply be a byproduct of the disease process and may not effectively alter its course. A recent discovery has now addressed this issue and has unequivocally put inflammation in general and specifically the microglial response center stage.

\section{Triggering Receptor Expressed on Myeloid Cells 2 (TREM2)}

TREM2 is expressed on the cell membrane of many types of immune cells including macrophages, dendritic cells, 
osteoclasts, and microglia [62]. TREM2 is thought to act as a cell surface receptor, and although the endogenous ligand has yet to be identified, it is known that it requires the adaptor protein 12 (DAP12) for the initiation of signaling cascades [63]. Activation of the TREM2 receptor on microglia has two important function consequences: (1) stimulation of phagocytosis activity and (2) decreased microglial proinflammatory responses [64]. Collectively, TREM2 may function to help aid microglia to clear damaged or apoptotic cells and cellular debris and help resolve damage-induced inflammation.

Insights into the important role that TREM2 plays have been deduced from individuals harboring homozygous mutations in the TREM2 gene. Such a mutation leads to The Nasu-Hakola disease, which manifests as a combination of bone cysts and dementia [65]. Affected individuals show progressive inflammatory neurodegeneration with loss of white matter and cystic bone lesions followed by death by the fifth decade of life [66].

Further support for TREM2 being an important mediator in neuroinflammation comes from animal models of multiple sclerosis (MS). Two different studies have reported a protective role of TREM2. In one study, blockade of TREM2 function enhanced disease progression in an experimental murine model of autoimmune encephalomyelitis [67]. In the second study, intravenous application of TREM2-transduced myeloid cells limited tissue destruction and facilitated repair in a murine model of MS [68]. Taken together, these studies highlight a critical role for TREM2 during inflammatory responses in the CNS.

\section{TREM2 in Alzheimer's Disease}

TREM2 function may affect AD pathology through phagocytosis. In a murine model of AD, Frank et al. demonstrated that TREM2 is upregulated in microglia found at the border of amyloid plaque deposits [69]. Moreover, TREM2 expression has been positively correlated with the phagocytic clearance of beta-amyloid in APP transgenic mice [70]. Given the well-documented role that microglia play in removing betaamyloid [61], the expression of TREM2 by beta-amyloid plaque-associated microglia may be interpreted as an effort to enhance beta-amyloid clearance and to limit the proinflammatory cytokine expression in response to microglia activation by beta-amyloid itself. Besides clearing beta-amyloid, TREM2 may also function to remove debris and participate in synapse remodeling [71].

The strongest evidence to date supporting a role for inflammation in AD comes from two recent studies demonstrating that TREM2 variants increase the risk for $\mathrm{AD}$ approximately 3 -fold $[72,73]$. These studies indicated that individuals that are heterozygous for several TREM2 mutations (the most common variant being a $\mathrm{R} 47 \mathrm{H}$ change) were at a greater risk for $\mathrm{AD}$. In addition, Jonsson and colleagues also showed that elderly carriers of the TREM2 variant who were asymptomatic for $\mathrm{AD}$, nevertheless, performed worse in cognitive exams as compared to noncarriers [72]. It is noteworthy that this particular mutation is extremely rare, only being found at a frequency of 0.63 percent. Compare this to the greatest risk factor for late-onset $\mathrm{AD}$, the apoE4 allele, in which it has been estimated that approximately $40 \%$ of $\mathrm{AD}$ subjects currently harbor at least one copy of this allele [74].

Interestingly, it is only individuals that are heterozygous for TREM2 that are at risk for AD: homozygous, autosomal recessive mutations for TREM2 result in The Nasu-Hakola disease in some individuals characterized by bone cysts and dementia [75], while resulting in fronttemporal dementia without bone disease in others [76]. Due to the common thread of dementia in these homozygous mutations, it was these initial findings that spurred further research to search for heterozygous mutations in the TREM2 gene in $\mathrm{AD}$ subjects.

Given the prophagocytic role of TREM2, loss of phagocytic activity of microglia could represent one mechanism by which the TREM2 mutations increased the risk to develop $\mathrm{AD}$ (Figure 3). Because TREM2 functions in microglia to also dampen microglial activation, mutations and loss of function could result in runaway inflammation as well. Based on these results, it is tempting to speculate on the potential pharmacological value that TREM2 agonists might have in AD. Future directions should answer this question as well as the possibility of finding more rare variants that have similar effects, including those in DAP12 and TREM2's intracellular signaling partner.

\section{Concluding Remarks}

Research in the field of $\mathrm{AD}$ has uncovered the detailed molecular mechanisms leading to the hallmark, microscopic lesions consisting of beta-amyloid plaques and NFTs. However, how these lesions lead to neurodegeneration is still under investigation. An important consideration in this paper was the attempt to unify the various potential players that contribute to cell death in $\mathrm{AD}$, including beta-amyloid, NFTs, caspases, oxidative stress, and inflammation. In this regard, it is suggested that beta-amyloid in the form of soluble beta-amyloid oligomers represents the earliest known step in the entire process, setting off a chain of events that ultimately lead to chronic inflammation and neuronal cell death. It has been difficult to assess the cause and effect relationship of inflammation in $\mathrm{AD}$, but the recent discovery of the TREM2 mutations has now put inflammation back on center stage as a process that contributes to disease progression. This is highlighted by the data indicating an approximate 3 -fold increase in the risk for $\mathrm{AD}$ in individuals harboring heterozygous variants in the TREM2 gene. Because TREM2 functions to modulate the inflammatory immune responses in microglia, mutations in this gene, in turn, could contribute to disease pathogenesis by preventing the clearance of beta-amyloid deposits and/or by enhancing inflammation. However, because the function of TREM2 is still poorly understood, it is difficult to determine how loss of TREM2 function might contribute to the disease process. Future studies examining a direct role for TREM2 in $\mathrm{AD}$ should help shed light on this question and provide further support for the role of chronic inflammation in this disease. 


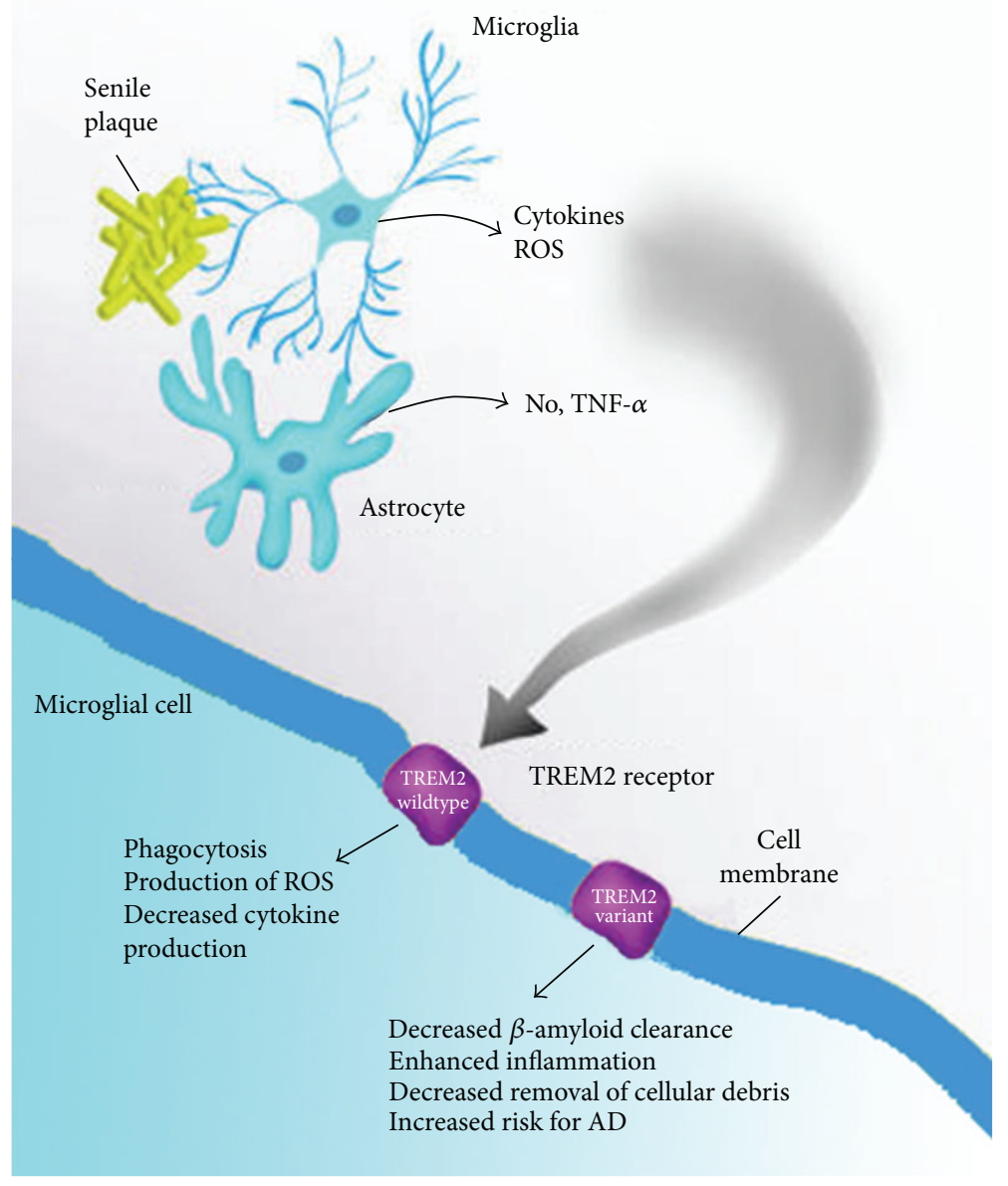

FIgURE 3: A central role for microglia in Alzheimer's disease is dependent upon a functional TREM2 receptor. Microglia represent one of the three classes of glia cells, whose primary function is to act as a major line of active immune defense in the CNS. In AD, microglia (top) and astrocytes (bottom) function in phagocytosis and in this regard help clear the brain of beta-amyloid deposits and apoptotic cells as well as any cellular debris. The important actions of microglia appear to be mediated through activation of the TREM2 receptor whose few known roles include suppressing inflammation and stimulating phagocytosis. As shown recently, variants in the TREM2 receptor have been discovered, and it has been suggested that the change in sequence leads to a loss of receptor function. It has been hypothesized that the loss of TREM2 activity has two major consequences: (1) decreased ability of microglia to remove extracellular deposits of beta-amyloid and (2) enhanced neuroinflammation. The loss of TREM2 function and altered immune responses by microglia may explain the increased risk for AD for individuals carrying the heterozygous mutations in TREM2.

\section{Abbreviations}

AD: Alzheimer's disease

apoE4: Apolipoprotein 4

APP: Amyloid precursor protein

NFTs: Neurofibrillary tangles

PHFs: Paired helical filaments

TREM2: Triggering receptor expressed on myeloid cells 2.

\section{Acknowledgments}

This work was funded by KO Dementia Foundation, Boise Idaho, and a NASA Grant NNX10AN29A. no. to the author.

\section{References}

[1] A. S. Association, "2012 Alzheimer's disease facts and figures," Alzheimer's \& Dementia, vol. 8, no. 2, pp. 131-168, 2012.
[2] B. L. Plassman, K. M. Langa, G. G. Fisher et al., "Prevalence of dementia in the United States: the aging, demographics, and memory study," Neuroepidemiology, vol. 29, no. 1-2, pp. 125-132, 2007.

[3] L. Alves, A. S. Correia, R. Miguel, P. Alegria, and P. Bugalho, "Alzheimer's disease: a clinical practice-oriented review," Frontiers in Neurology, vol. 3, p. 63, 2012.

[4] T. E. Golde, D. Dickson, and M. Hutton, "Filling the gaps in the $\mathrm{A} \beta$ cascade hypothesis of Alzheimer's disease," Current Alzheimer Research, vol. 3, no. 5, pp. 421-430, 2006.

[5] J. Hardy and D. J. Selkoe, "The amyloid hypothesis of Alzheimer's disease: progress and problems on the road to therapeutics," Science, vol. 297, no. 5580, pp. 353-356, 2002.

[6] S. T. Ferreira and W. L. Klein, "The Abeta oligomer hypothesis for synapse failure and memory loss in Alzheimer's disease," Neurobiology of Learning and Memory, vol. 96, no. 4, pp. 529$543,2011$. 
[7] C. A. McLean, R. A. Cherny, F. W. Fraser et al., "Soluble pool of Abeta amyloid as a determinant of severity of neurodegeneration in Alzheimer's disease," Annals of Neurology, vol. 46, no. 6, pp. 860-866, 1999.

[8] K. Broersen, F. Rousseau, and J. Schymkowitz, "The culprit behind amyloid beta peptide related neurotoxicity in Alzheimer's disease: oligomer size or conformation?" Alzheimer's Research \& Therapy, vol. 2, no. 4, p. 12, 2010.

[9] P. N. Lacor, M. C. Buniel, L. Chang et al., "Synaptic targeting by Alzheimer's-related amyloid $\beta$ oligomers," Journal of Neuroscience, vol. 24, no. 45, pp. 10191-10200, 2004.

[10] M. P. Lambert, A. K. Barlow, B. A. Chromy et al., "Diffusible, nonfibrillar ligands derived from A $\beta 1-42$ are potent central nervous system neurotoxins," Proceedings of the National Academy of Sciences of the United States of America, vol. 95, no. 11, pp. 6448-6453, 1998.

[11] D. D. Christensen, "Alzheimer's disease: progress in the development of anti-amyloid disease-modifying therapies," CNS Spectrums, vol. 12, no. 2, pp. 113-123, 2007.

[12] A. F. Teich and O. Arancio, "Is the amyloid hypothesis of Alzheimer's disease therapeutically relevant?" Biochemical Journal, vol. 446, no. 2, pp. 165-177, 2012.

[13] A. Extance, "Alzheimer's failure raises questions about diseasemodifying strategies," Nature Reviews Drug Discovery, vol. 9, no. 10, pp. 749-750, 2010.

[14] G. T. Bramblett, M. Goedert, R. Jakes, S. E. Merrick, J. Q. Trojanowski, and V. M. Y. Lee -, "Abnormal tau phosphorylation at Ser396 in Alzheimer's disease recapitulates development and contributes to reduced microtubule binding," Neuron, vol. 10, no. 6, pp. 1089-1099, 1993.

[15] J. Q. Trojanowski and V. M. Y. Lee, "Fatal Attractions' of proteins: a comprehensive hypothetical mechanism underlying Alzheimer's disease and other neurodegenerative disorders," Annals of the New York Academy of Sciences, vol. 924, pp. 6267, 2000.

[16] A. D. C. Alonso, T. Zaidi, M. Novak, I. Grundke-Iqbal, and K. Iqbal, "Hyperphosphorylation induces self-assembly of $\tau$ into tangles of paired helical filaments/straight filaments," Proceedings of the National Academy of Sciences of the United States of America, vol. 98, no. 12, pp. 6923-6928, 2001.

[17] H. G. Lee, G. Perry, P. I. Moreira et al., “Tau phosphorylation in Alzheimer's disease: pathogen or protector?" Trends in Molecular Medicine, vol. 11, no. 4, pp. 164-169, 2005.

[18] C. Reitz, "Alzheimer's disease and the amyloid cascade hypothesis: a critical review, International Journal of Alzheimer's Disease, vol. 2012, Article ID 369808, 11 pages, 2012.

[19] P. D. Coleman and P. J. Yao, "Synaptic slaughter in Alzheimer's disease," Neurobiology of Aging, vol. 24, no. 8, pp. 1023-1027, 2003.

[20] P. Coleman, H. Federoff, and R. Kurlan, "A focus on the synapse for neuroprotection in Alzheimer disease and other dementias," Neurology, vol. 63, no. 7, pp. 1155-1162, 2004.

[21] J. H. Su, A. J. Anderson, B. J. Cummings, and C. W. Cotman, "Immunohistochemical evidence for apoptosis in Alzheimer's disease," NeuroReport, vol. 5, no. 18, pp. 2529-2533, 1994.

[22] C. W. Cotman and A. J. Anderson, "A potential role for apoptosis in neurodegeneration and Alzheimer's disease," Molecular Neurobiology, vol. 10, no. 1, pp. 19-45, 1995.

[23] J. H. Su, K. E. Nichol, T. Sitch et al., "DNA damage and activated caspase-3 expression in neurons and astrocytes: evidence for apoptosis in frontotemporal dementia," Experimental Neurology, vol. 163, no. 1, pp. 9-19, 2000.
[24] C. Stadelmann, T. L. Deckwerth, A. Srinivasan et al., "Activation of caspase-3 in single neurons and autophagic granules of granulovacuolar degeneration in Alzheimer's disease: evidence for apoptotic cell death," American Journal of Pathology, vol. 155, no. 5, pp. 1459-1466, 1999.

[25] J. H. Su, M. Zhao, A. J. Anderson, A. Srinivasan, and C. W. Cotman, "Activated caspase-3 expression in Alzheimer's and aged control brain: correlation with Alzheimer pathology," Brain Research, vol. 898, no. 2, pp. 350-357, 2001.

[26] L. A. Selznick, D. M. Holtzman, B. H. Han et al., "In situ immunodetection of neuronal caspase-3 activation in Alzheimer disease," Journal of Neuropathology and Experimental Neurology, vol. 58, no. 9, pp. 1020-1026, 1999.

[27] T. T. Rohn, E. Head, W. H. Nesse, C. W. Cotman, and D. H. Cribbs, "Activation of caspase- 8 in the Alzheimer's disease brain," Neurobiology of Disease, vol. 8, no. 6, pp. 1006-1016, 2001.

[28] T. T. Rohn, R. A. Rissman, M. C. Davis, Y. E. Kim, C. W. Cotman, and E. Head, "Caspase-9 activation and caspase cleavage of tau in the Alzheimer's disease brain," Neurobiology of Disease, vol. 11, no. 2, pp. 341-354, 2002.

[29] A. LeBlanc, H. Liu, C. Goodyer, C. Bergeron, and J. Hammond, "Caspase-6 role in apoptosis of human neurons, amyloidogenesis, and Alzheimer's disease," Journal of Biological Chemistry, vol. 274, no. 33, pp. 23426-23436, 1999.

[30] H. Guo, S. Albrecht, M. Bourdeau, T. Petzke, C. Bergeron, and A. C. LeBlanc, "Active caspase-6 and caspase-6-cleaved tau in neuropil threads, neuritic plaques, and neurofibrillary tangles of Alzheimer's disease," American Journal of Pathology, vol. 165, no. 2, pp. 523-531, 2004.

[31] T. C. Gamblin, F. Chen, A. Zambrano et al., "Caspase cleavage of tau: linking amyloid and neurofibrillary tangles in Alzheimer's disease," Proceedings of the National Academy of Sciences of the United States of America, vol. 100, no. 17, pp. 10032-10037, 2003.

[32] R. A. Rissman, W. W. Poon, M. Blurton-Jones et al., "Caspasecleavage of tau is an early event in Alzheimer disease tangle pathology," Journal of Clinical Investigation, vol. 114, no. 1, pp. 121-130, 2004.

[33] F. G. Gervais, D. Xu, G. S. Robertson et al., "Involvement of caspases in proteolytic cleavage of Alzheimer's amyloid- $\beta$ precursor protein and amyloidogenic A $\beta$ peptide formation," Cell, vol. 97, no. 3, pp. 395-406, 1999.

[34] T. T. Rohn, E. Wirawan, R. J. Brown, J. R. Harris, E. Masliah, and P. Vandenabeele, "Depletion of Beclin-1 due to proteolytic cleavage by caspases in the Alzheimer's disease brain," Neurobiology of Disease, vol. 43, no. 1, pp. 68-78, 2011.

[35] P. E. Mouser, E. Head, K. H. Ha, and T. T. Rohn, "Caspasemediated cleavage of glial fibrillary acidic protein within degenerating astrocytes of the Alzheimer's disease brain," American Journal of Pathology, vol. 168, no. 3, pp. 936-946, 2006.

[36] T. L. Spires-Jones, A. De Calignon, T. Matsui et al., "In vivo imaging reveals dissociation between caspase activation and acute neuronal death in tangle-bearing neurons," Journal of Neuroscience, vol. 28, no. 4, pp. 862-867, 2008.

[37] K. Hensley, J. M. Carney, M. P. Mattson et al., "A model for $\beta$-amyloid aggregation and neurotoxicity based on free radical generation by the peptide: relevance to Alzheimer disease," Proceedings of the National Academy of Sciences of the United States of America, vol. 91, no. 8, pp. 3270-3274, 1994.

[38] S. Varadarajan, S. Yatin, M. Aksenova, and D. A. Butterfield, "Review: Alzheimer's amyloid $\beta$-peptide-associated free radical oxidative stress and neurotoxicity," Journal of Structural Biology, vol. 130, no. 2-3, pp. 184-208, 2000. 
[39] D. A. Butterfield, "Amyloid $\beta$-peptide (1-42)-induced oxidative stress and neurotoxicity: implications for neurodegeneration in Alzheimer's disease brain. A review," Free Radical Research, vol. 36, no. 12, pp. 1307-1313, 2002.

[40] M. G. Bartley, K. Marquardt, D. Kirchhof, H. M. Wilkins, D. Patterson, and D. A. Linseman, "Overexpression of amyloid-beta protein precursor induces mitochondrial oxidative stress and activates the intrinsic apoptotic cascade," Journal of Alzheimer's Disease, vol. 28, no. 4, pp. 855-868, 2012.

[41] Z. Cai, B. Zhao, and A. Ratka, "Oxidative stress and betaamyloid protein in Alzheimer's disease," NeuroMolecular Medicine, vol. 13, no. 4, pp. 223-250, 2011.

[42] A. Gómez-Ramos, J. Díaz-Nido, M. A. Smith, G. Perry, and J. Avila, "Effect of the lipid peroxidation product acrolein on tau phosphorylation in neural cells," Journal of Neuroscience Research, vol. 71, no. 6, pp. 863-870, 2003.

[43] A. Takeda, M. A. Smith, J. Avilá et al., "In Alzheimer's disease, heme oxygenase is coincident with Alz50, an epitope of $\tau$ induced by 4 -hydroxy-2-nonenal modification," Journal of Neurochemistry, vol. 75, no. 3, pp. 1234-1241, 2000.

[44] N. Y. Calingasan, K. Uchida, and G. E. Gibson, "Protein-bound acrolein: a novel marker of oxidative stress in Alzheimer's disease," Journal of Neurochemistry, vol. 72, no. 2, pp. 751-756, 1999.

[45] M. Pérez, R. Cuadros, M. A. Smith, G. Perry, and J. Avila, "Phosphorylated, but not native, tau protein assembles following reaction with the lipid peroxidation product, 4-hydroxy-2nonenal," FEBS Letters, vol. 486, no. 3, pp. 270-274, 2000.

[46] X. Zhu, C. A. Rottkamp, H. Boux, A. Takeda, G. Perry, and M. A. Smith, "Activation of p38 kinase links tau phosphorylation, oxidative stress, and cell cycle-related events in Alzheimer disease," Journal of Neuropathology and Experimental Neurology, vol. 59, no. 10, pp. 880-888, 2000.

[47] X. Zhu, R. J. Castellani, A. Takeda et al., "Differential activation of neuronal ERK, JNK/SAPK and p38 in Alzheimer disease: the "two hit" hypothesis," Mechanisms of Ageing and Development, vol. 123, no. 1, pp. 39-46, 2001.

[48] L. F. Lue, L. Brachova, W. H. Civin, and J. Rogers, "Inflammation, $\mathrm{A} \beta$ deposition, and neurofibrillary tangle formation as correlates of Alzheimer's disease neurodegeneration," Journal of Neuropathology and Experimental Neurology, vol. 55, no. 10, pp. 1083-1088, 1996.

[49] J. Rogers, S. Webster, L. F. Lue et al., "Inflammation and Alzheimer's disease pathogenesis," Neurobiology of Aging, vol. 17, no. 5, pp. 681-686, 1996.

[50] P. L. McGeer, S. Itagaki, H. Tago, and E. G. McGeer, "Reactive microglia in patients with senile dementia of the Alzheimer type are positive for the histocompatibility glycoprotein HLA-DR," Neuroscience Letters, vol. 79, no. 1-2, pp. 195-200, 1987.

[51] C. E. Shepherd, E. Thiel, H. McCann, A. J. Harding, and G. M. Halliday, "Cortical inflammation in Alzheimer disease but not dementia with Lewy bodies," Archives of Neurology, vol. 57, no. 6, pp. 817-822, 2000.

[52] J. Tan, T. Town, D. Paris et al., "Microglial activation resulting from CD40-CD401 interaction after $\beta$ - amyloid stimulation," Science, vol. 286, no. 5448, pp. 2352-2355, 1999.

[53] M. Johnstone, A. J. H. Gearing, and K. M. Miller, "A central role for astrocytes in the inflammatory response to $\beta$ - amyloid; chemokines, cytokines and reactive oxygen species are produced," Journal of Neuroimmunology, vol. 93, no. 1-2, pp. 182193, 1999.
[54] J. C. S. Breitner, "Inflammatory processes and antiinflammatory drugs in Alzheimer's disease: a current appraisal," Neurobiology of Aging, vol. 17, no. 5, pp. 789-794, 1996.

[55] J. Rogers, J. Luber-Narod, S. D. Styren, and W. H. Civin, "Expression of immune system-associated antigens by cells of the human central nervous system: relationship to the pathology of Alzheimer's disease," Neurobiology of Aging, vol. 9, no. 4, pp. 339-349, 1988.

[56] T. Wyss-Coray and J. Rogers, "Inflammation in Alzheimer disease-a brief review of the basic science and clinical literature," Cold Spring Harbor Perspectives in Medicine, vol. 2, no. 1, p. a006346, 2012.

[57] T. Wyss-Coray, "Inflammation in Alzheimer disease: driving force, bystander or beneficial response?" Nature Medicine, vol. 12, no. 9, pp. 1005-1015, 2006.

[58] S. Itagaki, P. L. McGeer, H. Akiyama, S. Zhu, and D. Selkoe, "Relationship of microglia and astrocytes to amyloid deposits of Alzheimer disease," Journal of Neuroimmunology, vol. 24, no. 3, pp. 173-182, 1989.

[59] Q. Wu, C. Combs, S. B. Cannady, D. S. Geldmacher, and K. Herrup, "Beta-amyloid activated microglia induce cell cycling and cell death in cultured cortical neurons," Neurobiology of Aging, vol. 21, no. 6, pp. 797-806, 2000.

[60] L. Meda, P. Baron, E. Prat et al., "Proinflammatory profile of cytokine production by human monocytes and murine microglia stimulated with $\beta$-amyloid[25-35]," Journal of Neuroimmunology, vol. 93, no. 1-2, pp. 45-52, 1999.

[61] C. Y. D. Lee and G. E. Landreth, "The role of microglia in amyloid clearance from the AD brain," Journal of Neural Transmission, vol. 117, no. 8, pp. 949-960, 2010.

[62] M. Colonna, "Trems in the immune system and beyond," Nature Reviews Immunology, vol. 3, no. 6, pp. 445-453, 2003.

[63] A. Bouchon, J. Dietrich, and M. Colonna, "Cutting edge: inflammatory responses can be triggered by TREM-1, a novel receptor expressed on neutrophils and monocytes," Journal of Immunology, vol. 164, no. 10, pp. 4991-4995, 2000.

[64] K. Takahashi, C. D. P. Rochford, and H. Neumann, "Clearance of apoptotic neurons without inflammation by microglial triggering receptor expressed on myeloid cells-2," Journal of Experimental Medicine, vol. 201, no. 4, pp. 647-657, 2005.

[65] J. Paloneva, M. Kestilä, J. Wu et al., "Loss-of-function mutations in TYROBP (DAP12) result in a presenile dementia with bone cysts," Nature Genetics, vol. 25, no. 3, pp. 357-361, 2000.

[66] J. Paloneva, J. Mandelin, A. Kiialainen et al., "DAP12/TREM2 deficiency results in impaired osteoclast differentiation and osteoporotic features," Journal of Experimental Medicine, vol. 198, no. 4, pp. 669-675, 2003.

[67] L. Piccio, C. Buonsanti, M. Mariani et al., "Blockade of TREM2 exacerbates experimental autoimmune encephalomyelitis," European Journal of Immunology, vol. 37, no. 5, pp. 1290-1301, 2007.

[68] K. Takahashi, M. Prinz, M. Stagi, O. Chechneva, and H. Neumann, “TREM2-transduced myeloid precursors mediate nervous tissue debris clearance and facilitate recovery in an animal model of multiple sclerosis," PLoS Medicine, vol. 4, no. 4, pp. 675-689, 2007.

[69] S. Frank, G. J. Burbach, M. Bonin et al., "TREM2 is upregulated in amyloid plaque-associated microglia in aged APP23 transgenic mice," Glia, vol. 56, no. 13, pp. 1438-1447, 2008.

[70] B. Melchior, A. E. Garcia, B. K. Hsiung et al., "Dual induction of TREM2 and tolerance-related transcript, Tmem176b, in amyloid transgenic mice: implications for vaccine-based therapies 
for Alzheimer's disease," ASN Neuro, vol. 2, no. 3, pp. 157-170, 2010.

[71] M. E. Tremblay, R. L. Lowery, and A. K. Majewska, "Microglial interactions with synapses are modulated by visual experience," PLoS Biology, vol. 8, no. 11, Article ID e1000527, 2010.

[72] T. Jonsson, H. Stefansson, D. S. Ph et al., "Variant of TREM2 associated with the risk of Alzheimer's disease," The New England Journal of Medicine, vol. 368, pp. 107-116, 2013.

[73] R. Guerreiro, A. Wojtas, J. Bras et al., "TREM2 variants in Alzheimer's disease," The New England Journal of Medicine, vol. 368, pp. 117-127, 2013.

[74] M. Eisenstein, "Genetics: finding risk factors," Nature, vol. 475, no. 7355, pp. S20-S22, 2011.

[75] J. Paloneva, T. Manninen, G. Christman et al., "Mutations in two genes encoding different subunits of a receptor signaling complex result in an identical disease phenotype," American Journal of Human Genetics, vol. 71, no. 3, pp. 656-662, 2002.

[76] R. J. Guerreiro, E. Lohmann, J. M. Bras et al., "Using exome sequencing to reveal mutations in TREM2 presenting as a frontotemporal dementia-like syndrome without bone involvement," Archives of Neurology, pp. 1-7, 2012. 


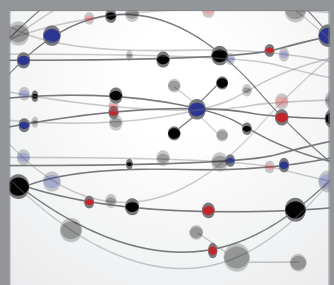

The Scientific World Journal
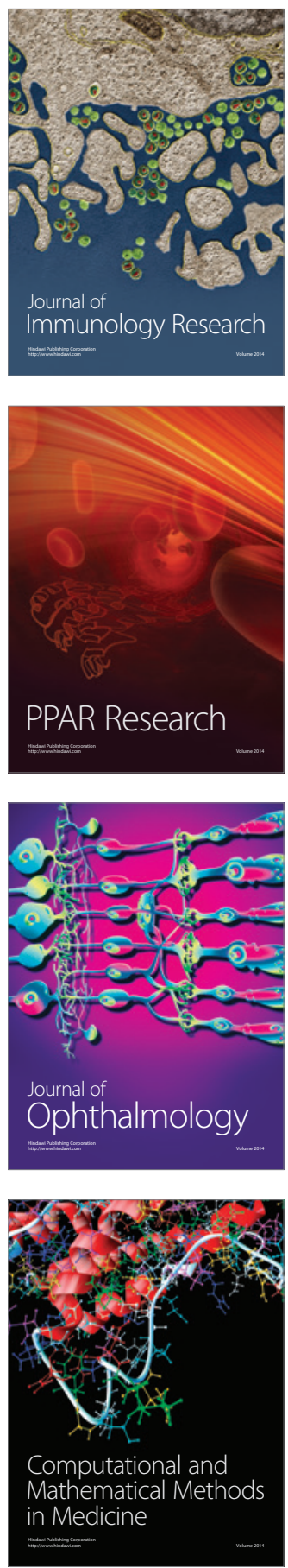

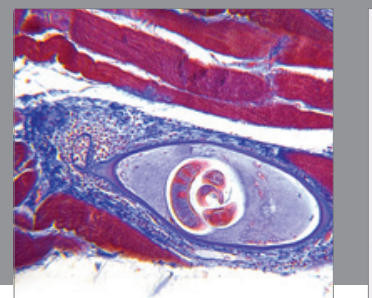

Gastroenterology

Research and Practice
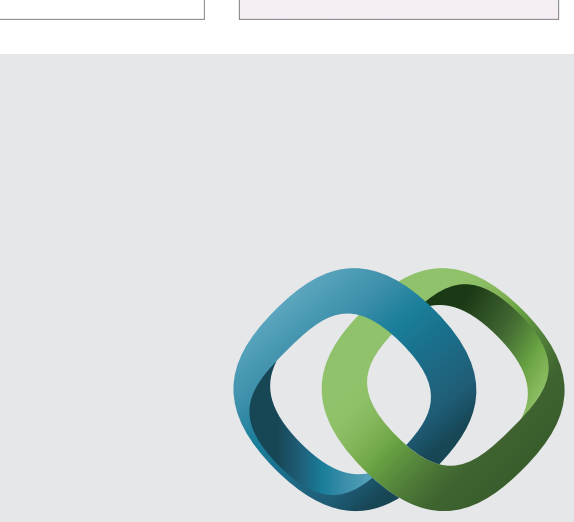

\section{Hindawi}

Submit your manuscripts at

http://www.hindawi.com
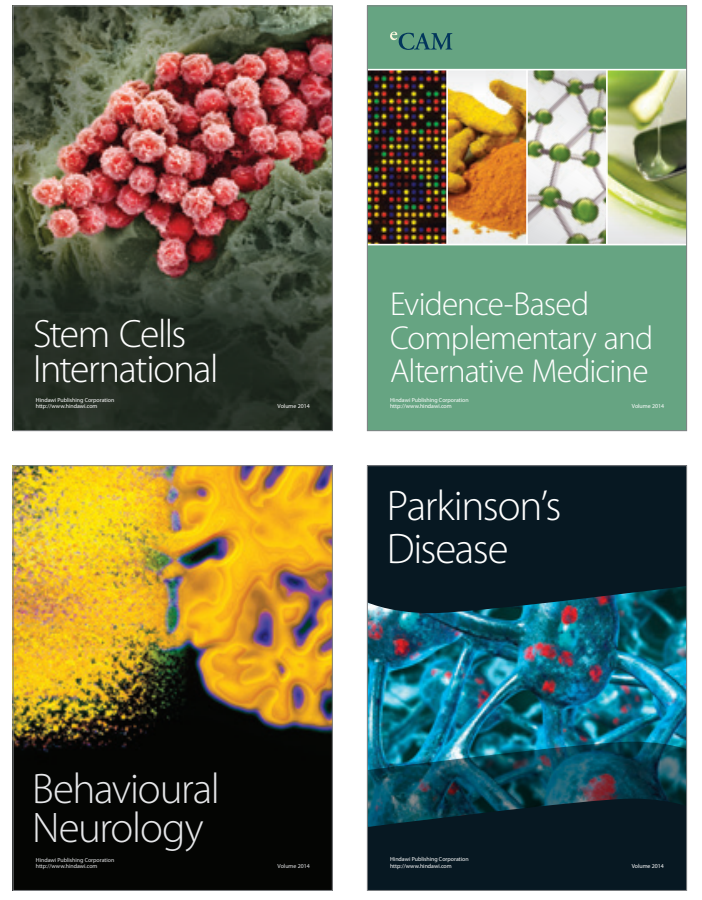
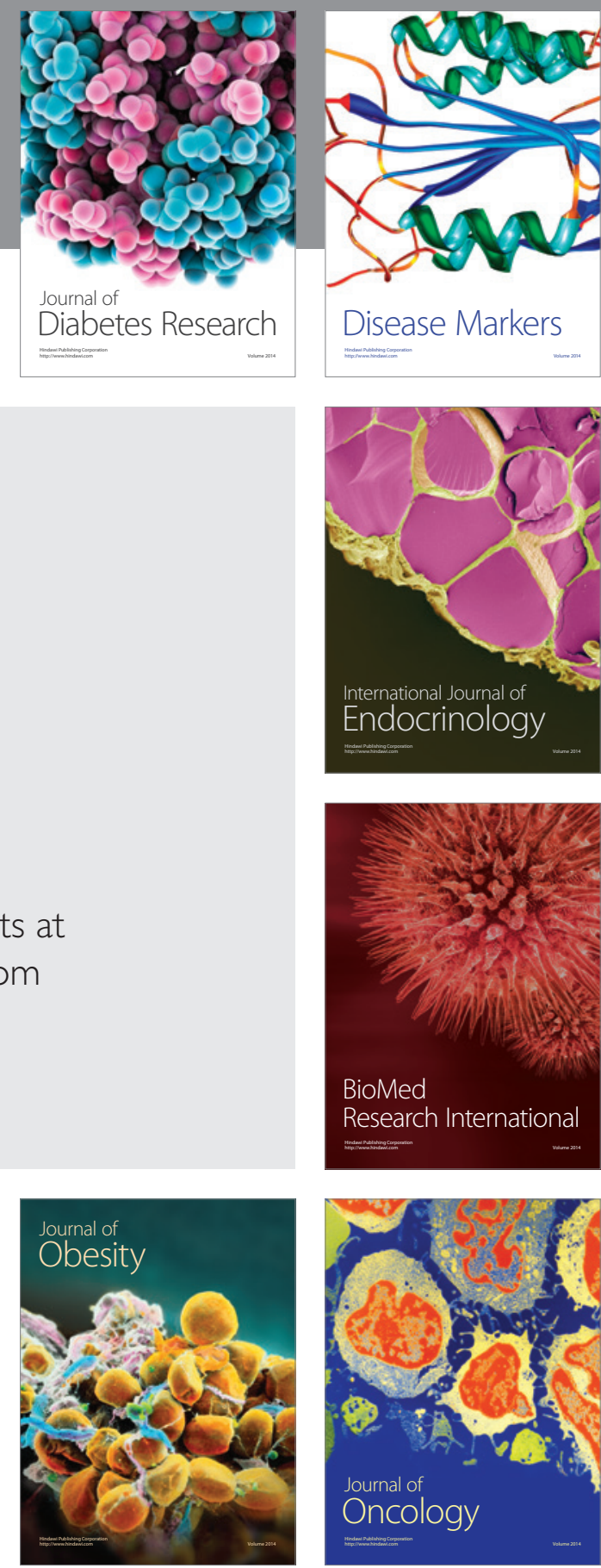

Disease Markers
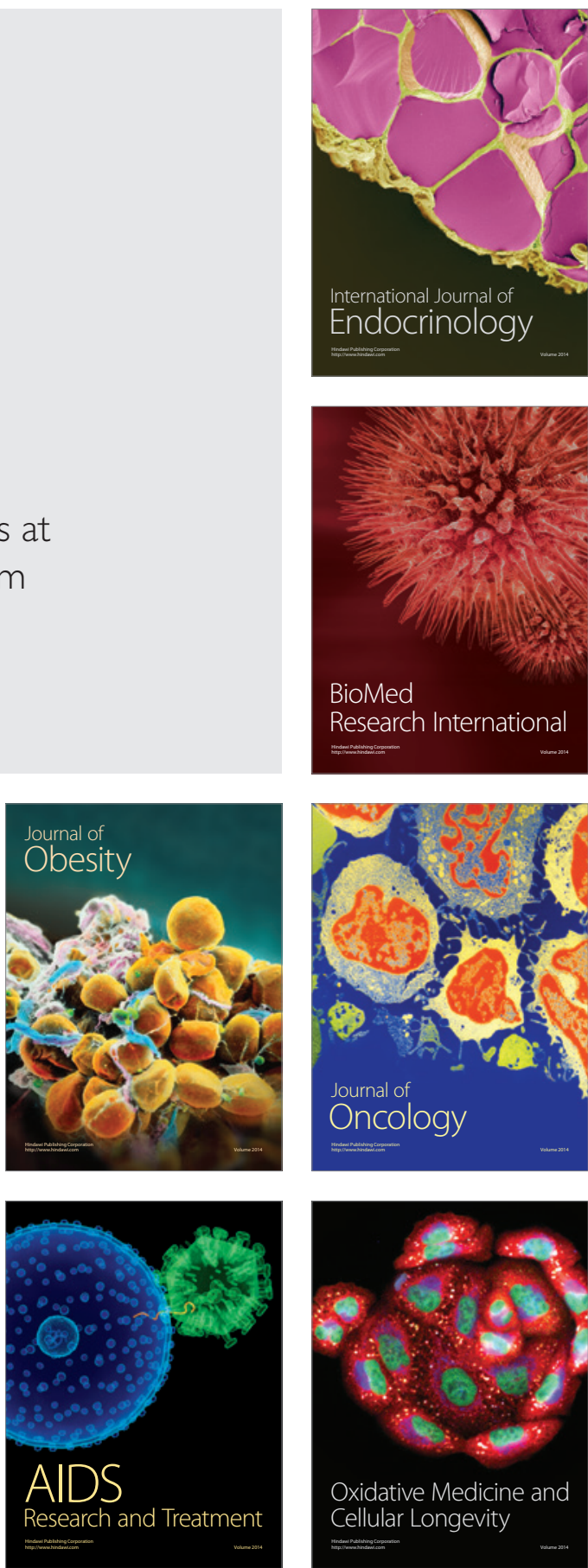